\title{
Space group symmetry applied to SCF calculations with periodic boundary conditions and Gaussian orbitals
}

\author{
Alexander A. Rusakov, ${ }^{1}$ Michael J. Frisch, ${ }^{2}$ and Gustavo E. Scuseria ${ }^{1,3}$ \\ ${ }^{1}$ Department of Chemistry, Rice University, Houston, Texas 77005, USA \\ ${ }^{2}$ Gaussian, Inc., 340 Quinnipiac Street, Building 40, Wallingford, Connecticut 06492, USA \\ ${ }^{3}$ Department of Physics and Astronomy, Rice University, Houston, Texas 77005, USA
}

(Received 29 May 2013; accepted 3 September 2013; published online 19 September 2013)

\begin{abstract}
Space group symmetry is exploited and implemented in density functional calculations of extended systems with periodic boundary conditions. Our scheme for reducing the number of two-electron integrals employs the entire set of operations of the space group, including glide plains and screw axes. Speedups observed for the Fock matrix formation in simple 3D systems range from $2 \mathrm{X}$ to 9X for the near field Coulomb part and from $3 \mathrm{X}$ to $8 \mathrm{X}$ for the Hartree-Fock-type exchange, the slowest steps of the procedure, thus leading to a substantial reduction of the computational time. The relatively small speedup factors in special cases are attributed to the highly symmetric positions atoms occupy in crystals, including the ones tested here, as well as to the choice of the smallest possible unit cells. For quasi-1D systems with most atoms staying invariant only under identity, the speedup factors often exceed one order of magnitude reaching almost 70X (near-field Coulomb) and 57X (HFx) for the largest tested $(16,7)$ single-walled nanotube with 278 symmetry operations. (C) 2013 AIP Publishing LLC. [http://dx.doi.org/10.1063/1.4821352]
\end{abstract}

\section{INTRODUCTION}

Effective one-electron approximations, including density functional theory (DFT) in the Kohn-Sham formulation, remain among the most popular and powerful tools for $a b$ initio modeling of extended systems, including formally infinite systems with periodic boundary conditions (PBC). ${ }^{1}$ Of special importance are orbital-dependent and primarily hybrid functionals containing a fraction of exact Hartree-Fock-type exchange (HFx), ${ }^{2}$ as they are found to generally lead to better levels of accuracy for a broad class of systems including $3 \mathrm{D}$ crystals where screening of the Coulomb potential in exchange interactions yields significantly more accurate results. ${ }^{3,4}$ Efficient techniques to attain linear scaling of the computation time with respect to the size of the system have been developed and implemented for the far-field Coulomb ${ }^{5}$ and exchange-correlation parts ${ }^{6}$ of the Kohn-Sham procedure. The relative increase of the computational cost upon incorporating HFx remains modest for molecules, but becomes noticeable for $1 \mathrm{D}$ and especially pronounced for 3D periodic systems. Efficient approaches based on the Ewald potential function $^{7}$ and fast multipole method (FMM) ${ }^{8}$ cannot be directly applied to attain linear scaling of HFx, though other sophisticated screening techniques enable it. ${ }^{9}$ Nevertheless, the computation cost associated with the large number of explicitly evaluated two-electron integrals can easily get demanding for systems with large unit cells, e.g., structures with defects or chiral nanotubes. Exploiting full space group symmetry of infinite systems can substantially reduce the number of required two-electron integrals and noticeably save computational time of the most expensive HFx step, as well as for the near-field Coulomb matrix evaluation.

Throughout this work, we will be referring to the electronic (non-relativistic) Hamiltonian expressed in the basis of Cartesian Gaussian type orbitals. This discretization is advantageous primarily due to the ease of analytically evaluating molecular integrals. The localized form of Gaussian basis functions also plays a key role in screening procedures.

\section{THEORETICAL BACKGROUND}

Three-dimensional structures periodic in 1, 2, and 3 dimensions are described by line, layer, and space groups, respectively. ${ }^{10,11}$ Our discussion will be mostly focused on the general case of systems periodic in 3 dimensions. There are totally 230 space groups, an arbitrary operation whereof has the form $(\mathbf{R} \mid \mathbf{t})$, where $\mathbf{R}$ is a $3 \times 3$ rotation matrix and $\mathbf{t}$ is a displacement vector. The latter can be different from a pure translation or zero vector for the nonsymmorphic groups which have screw axes and glide planes: these operations include a shift by a fraction of the primitive translation vector. The action of $(\mathbf{R} \mid \mathbf{t})$ on an arbitrary point $\mathbf{r}$ is given by

$$
\mathbf{r}_{1}=(\mathbf{R} \mid \mathbf{t}) \mathbf{r}=\mathbf{R r}+\mathbf{t} .
$$

The two points $\mathbf{r}$ and $\mathbf{r}_{\mathbf{1}}$ are deemed equivalent, $\mathbf{r} \sim \mathbf{r}_{\mathbf{1}}$, and so are two identical one-electron basis functions centered on point nuclei equivalent under a given operation.

For the molecular case, it has been shown ${ }^{12}$ that the Fock matrix $\mathbf{F}$ possesses the symmetry of the molecule. Thus, if $\mathbf{R}$ is a symmetry operation of the molecular point group and $\overline{\mathbf{R}}$ is the corresponding transformation of the basis set $\left(\phi_{i}\right.$, $i=\overline{1, n}$, then

$$
\mathbf{F}=\overline{\mathbf{R}}^{\dagger} \mathbf{F} \overline{\mathbf{R}} \text {. }
$$

Analogously to equivalent individual basis functions, one can introduce equivalent pairs and quartets of those which will consist of identical basis functions centered 
on equivalent nuclei, i.e., $\left(\phi_{i}\left[\mathbf{r}_{1}\right] \phi_{j}\left[\mathbf{r}_{2}\right]\right) \sim\left(\phi_{i}\left[\overline{\mathbf{r}}_{1}\right] \phi_{j}\left[\overline{\mathbf{r}}_{2}\right]\right)$ and $\left(\phi_{i}\left[\mathbf{r}_{1}\right] \phi_{j}\left[\mathbf{r}_{2}\right] \phi_{k}\left[\mathbf{r}_{3}\right] \phi_{l}\left[\mathbf{r}_{4}\right]\right) \sim\left(\phi_{i}\left[\overline{\mathbf{r}}_{1}\right] \phi_{j}\left[\overline{\mathbf{r}}_{2}\right] \phi_{k}\left[\overline{\mathbf{r}}_{3}\right] \phi_{l}\left[\overline{\mathbf{r}}_{4}\right]\right)$ if $\mathbf{r}_{m} \sim \overline{\mathbf{r}}_{m}, m=\overline{1,4}$. The fundamental result of Ref. 12, which plays a key role in our further discussion, is that the full two-electron contribution $\mathbf{G}$ to the Fock matrix $\mathbf{F}$ can be obtained by symmetrizing the skeleton two-electron matrix $\mathbf{G}_{s k}$. This skeleton matrix is calculated using only those two-electron integrals which result from the list of symmetry unique quartets of basis functions. The symmetrization step calculates the following sum where $i$ runs over all group operations:

$$
\mathbf{G}=\sum_{i=1}^{n} \overline{\mathbf{R}}_{i}^{\dagger} \mathbf{G}_{s k} \overline{\mathbf{R}}_{i} .
$$

Symmetrization of the Coulomb and exchange contributions is independent, thus making this technique applicable not only to the Hartree-Fock, but also to the Fock type matrix in the Kohn-Sham formulation of DFT.

For systems with periodic boundary conditions, Bloch sums are formed to comply with translational symmetry. The basis set of the Bloch sums enables decoupling of the Hartree-Fock type equations in reciprocal, or k-space. Evaluation of Fock type matrix elements in the basis of Bloch sums results in special kind of Fourier transformation for the Fock type matrix in direct space: ${ }^{5}$

$$
\mathbf{F}_{M N}^{\mathbf{k}}=\sum_{\mathbf{g}} \mathbf{F}_{\mu \nu}^{\mathbf{0 g}} \exp (i \mathbf{k} \cdot \mathbf{g}),
$$

where $\mu, v$ stand for the basis functions yielding the Bloch sums $M$ and $N$, and $\mathbf{g}$ is the direct space lattice vector formally running over the infinite crystal. In practical calculations, the range of $\mathbf{g}$ is limited to a finite portion of the crystal. ${ }^{9}$ Evaluation of the elements $\mathbf{F}_{\mu \nu}^{\mathbf{0 g}}$, where $\mu$ is always centered in the central cell and $v$ in some arbitrary cell (including the central one), is typically the most time-consuming step of the calculation, especially for hybrid functionals. Therefore, one can expect substantial reduction of the entire computation time upon applying symmetry in calculating the direct space Fock type matrix.

The derivation of the method in Ref. 12 does not necessarily imply that the pairs $\left(\phi_{i}\left[\mathbf{r}_{1}\right] \phi_{j}\left[\mathbf{r}_{2}\right]\right)$ forming the quartets are taken from the same set. A closer inspection of the expressions for the Coulomb and exchange contributions

$$
\begin{aligned}
& \mathbf{J}_{\mu \nu}^{\mathbf{0 g}}=\frac{1}{2} \sum_{\mathbf{m}, \mathbf{n}, \lambda, \sigma}\left(\mu_{0} v_{g} \mid\left(\lambda_{0} \sigma_{n}\right)_{m}\right) \mathbf{P}_{\lambda \sigma}^{\mathbf{0 n}}, \\
& \mathbf{K}_{\mu \nu}^{\mathbf{0 g}}=\sum_{\mathbf{m}, \mathbf{n}, \lambda, \sigma}\left(\mu_{0} \lambda_{m} \mid\left(v_{0} \sigma_{n}\right)_{g}\right) \mathbf{P}_{\lambda \sigma}^{\mathbf{0}, \mathbf{n}-\mathbf{m}}
\end{aligned}
$$

shows that the only difference of the right-hand side of the two-electron integrals (here presented in Mulliken notation) from the molecular case consists in the shift of the whole pair of basis functions by a translation vector (translational invariance of the density matrix was tacitly assumed in Eqs. (5) and (6)). Since in the formally infinite crystal all shifts are permissible, the right-hand sides form a closed set under the symmetry operations mapping the crystal onto itself, therefore validating the applicability of the basic statements of Ref. 12 to
$\mathbf{F}_{\mu \nu}^{0 \mathrm{~g}}$. Choosing the list of the unique quartets for the periodic case according to the technique proposed in Ref. 12 and further developed in Refs. 13 and 14 has a number of subtleties worth elucidating.

The set of applicable symmetry operations upon imposing periodic boundary conditions emerges naturally for symmorphic groups. If $\mathbf{G} / \mathbf{T}$ is a factor-group, where $\mathbf{T}$ is the normal subgroup of pure translations, the coset representatives can be chosen to have trivial translational part, i.e., in the form $(\mathbf{R} \mid \mathbf{0})$. These representatives form a group and can be directly applied. The translational component cannot be eliminated in the coset representatives if $\mathbf{G}$ is non-symmorphic. Regardless of the choice, the coset representatives do not form a group, since their products will generate full lattice translations not present in the set. Nevertheless, since the corresponding symmetry operations are applied to objects-two-electron integrals and Fock-type matrices-which are translationally invariant with respect to $\mathbf{T}$, the corresponding transformation matrices are devoid of pure translations and form a group. ${ }^{15}$ This allows to use operations corresponding to screw axes and glide plains similarly to the point group operations. Since, according to Secs. 2.11 and 11.1.1 of the International Tables for Crystallography, vol. A, ${ }^{10}$ the coordinate triplets of the general position (reduced "modulo 1") represent the symmetry operations of the space group in the matrix form, such matrices are convenient to take as symmetry transformations. Let us notice that for centered lattices the number of symmetry operations considered is $N+1$ times larger ( $N$ is the number of centering vectors) than the order of the factor-group thus yielding the complete general position explicitly.

Due to the format of the direct space Fock type matrix and the common convention of the lower-diagonal storage, two restrictions are imposed. First, one basis function must be retained within the central cell upon any operation. Second, $\bar{\mu} \geq \bar{v}$, where $\bar{\mu}$ and $\bar{v}$ are symmetry images of $\mu$ and $v$, respectively. In general, these conditions may not be satisfied automatically. If the first condition is not fulfilled, and the image $\bar{\mu}$ appears in some cell $\mathbf{g} \neq \mathbf{0}$, then, due to translation invariance of integrals and densities, the pair can be translated by the lattice vector $-\mathbf{g}$. For the second condition the right mapping is achieved by noting that $\mathbf{F}_{\mu \nu}^{\mathbf{0 g}}=\mathbf{F}_{v \mu}^{\mathbf{0},-\mathbf{g}}$. We address this in detail in Sec. III.

\section{METHOD AND IMPLEMENTATION}

Below we propose a detailed scheme for rejecting symmetry-equivalent two-electron integrals, building the skeleton Fock-type matrix and expanding it into the full one. Our approach is aimed at exploiting the full space-group symmetry of a given crystalline system to attain reduction of the SCF cycle computational cost.

\section{A. Selection of reduced list of shell quartets}

The reduced list of shell quartets naturally emerges if symmetry-unique left-hand sides - the unshifted pairs - are coupled with all possible right-hand sides-shifted pairsor vice versa. The former approach has a straightforward 
implementation since the requirement that one shell always resides in the reference cell assures the existence of a universal reduced list upon application of all space group operations. It appears not to be the case for the right-hand sides, as we will discuss later on.

Let $I^{0}$ represent the index of the shell $I$ in the central cell, $J^{g}$ - the index of the shell $J$ in an arbitrary cell $\mathbf{g}, N$ is the total number of shells within single unit cell. Then the algorithm of rejecting symmetry equivalent unshifted shell pairs can be organized as follows.

\section{Algorithm 1}

1. Ascribe a unique index to every $\left(I^{0} J^{g}\right)$-pair, e.g.,

$$
\text { Index }=I(I-1) / 2+J+g N(N+1) / 2 .
$$

2. For every $\left(I^{0} J^{g}\right)$-pair, loop over all symmetry operations and form symmetry images $\left(I^{\prime h} J^{\prime g^{\prime}}\right)$. One should notice that $I^{\prime}$ acquires a cell index $h$ as the nucleus carrying the shell may not necessarily stay within the reference cell upon applying a symmetry operation, as shown in Fig. 1.

If $h \neq 0$, then both shells $I^{\prime}$ and $J^{\prime}$ are shifted by a cell vector $\mathbf{- h}$ corresponding to the cell with index $h$ thus yielding $\left(I^{\prime 0} J^{\prime g^{\prime \prime}}\right), \mathbf{g}^{\prime \prime}=\mathbf{g}^{\prime}-\mathbf{h}$. Let us note that this situation occurs even for operations of symmorphic groups like simple 4-fold axis (in centered as well as primitive lattices), as illustrated. At the same time the imposed format restriction allows one to use operations of the non-symmorphic groups the same way as for the symmorphic ones.

If $I^{\prime}<J^{\prime}$, the equivalent pair is $\left(J^{\prime 0} I^{\prime g^{\prime \prime \prime}}\right), \mathbf{g}^{\prime \prime \prime}=-\mathbf{g}^{\prime \prime}$.

3. Calculate the Index $x^{\prime}$ of the shifted pair using Eq. (7). If Index $x^{\prime}>$ Index, remove the $\left(I^{0} J^{g}\right)$ pair from the list and continue with the next pair, otherwise keep it. If $M$ operations of the space group map the retained pair onto itself, Index ${ }^{\prime}=$ Index, ascribe it the weight factor $1 / M$.

Algorithm 1 is here always applied to computing the near-field Coulomb matrix due to certain convenience in matching it with the far-field Coulomb part evaluation based on FMM.

Atomic positions in the reference cell often have multiplicities less than the number of symmetry operations, i.e., their site-symmetry groups (Sec. 3.4 in Ref. 16) are nontrivial, and there is at least one operation, rather than identity, that leaves the given point invariant (in our case this can also result upon applying a shift, if needed). If the multiplicity of such position is low, the unshifted pairs list reduction can

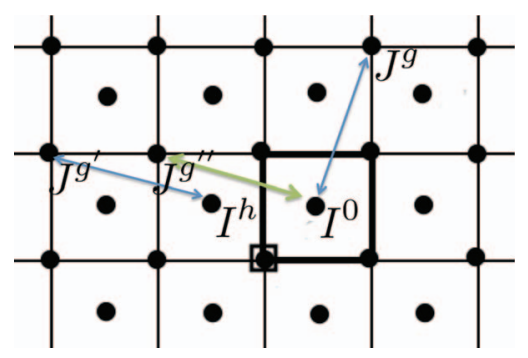

FIG. 1. Equivalence of shell pairs. $\left(I^{h} J^{g \prime}\right)$ results from a $\pi / 2$ rotation of $\left(I^{0} J^{g}\right)$, but should be shifted by as a whole by $-\mathbf{h}$ to yield $\left(I^{0} J^{g^{\prime} \prime}\right)$.

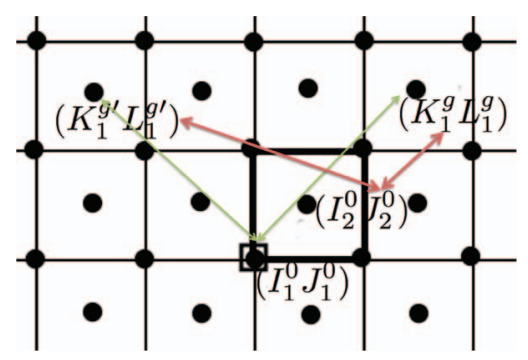

FIG. 2. On existence of unique right-hand side pairs. Pairs $\left(K_{1}^{g} L_{1}^{g}\right)$ and $\left(K_{1}^{g \prime} L_{1}^{g \prime}\right)$ are equivalent with respect to $\left(I_{1}^{0} J_{1}^{0}\right)$, but not with respect to $\left(I_{2}^{0} J_{2}^{0}\right)$.

be noticeably restricted. The alternative approach consists in applying symmetry to the shifted pairs, since the special position of the shifted atom is of higher (or same) multiplicity than that of the unshifted one. The shortcoming of this approach is that in general it is impossible to form a universal list of symmetry-unique right-hand sides. Fig. 2 illustrates the problem for the case of a square lattice with atoms in the corners and in the middle of the cell.

For simplicity, in Fig. 2 we take only the pairs of basis functions sharing the same nuclei. A 4-fold rotational axis passing through the origin is taken into account. The shell pair $\left(I_{1}^{0} J_{1}^{0}\right)$ at the origin remains intact under all rotations by $\pi / 2$. The images of the pair $\left(I_{2}^{0} J_{2}^{0}\right)$ are required to be brought back to the central cell, therefore the shell pair $\left(I_{2}^{0} J_{2}^{0}\right)$ also stays intact. The pairs $\left(K_{1}^{g} L_{1}^{g}\right)$ and $\left(K_{1}^{g \prime} L_{1}^{g \prime}\right)$ denote symmetry images of the shifted pair $\left(I_{1}^{0} J_{1}^{0}\right)$ upon applying the operations of the 4-fold axis. As it can be seen, the pairs $\left(K_{1}^{g} L_{1}^{g}\right)$ and $\left(K_{1}^{g \prime} L_{1}^{g \prime}\right)$ remain symmetrically equivalent with respect to the unshifted pair $\left(I_{1}^{0} J_{1}^{0}\right)$, but they do not do so with respect to the pair $\left(I_{2}^{0} J_{2}^{0}\right)$. This statement holds true for any case when at least one symmetry operation moves at least one nucleus form the reference cell outside its boundaries.

The reduction of the list of shifted pairs remains possible if there is a non-trivial subset $\mathbf{G}^{\prime}$ (still applied as described in Sec. II) of operations which leave all nuclei of the reference cell within this cell. Let us notice that such subset may still contain screw axes and glide plains. For this case we suggest the following algorithm.

\section{Algorithm 2}

1. Perform the left-hand side reduction as in Algorithm 1.

2. Form the initial list of the right-hand sides by applying all lattice translations to the reduced list of the left-hand sides.

3. Loop over the index of the retained pairs. If the pair is retained with the weigh factor 1 , further reduction is impossible, and the pair should be left intact for all shifts.

4. If a pair is retained with a fractional weight, then loop over all shifts.

5. Apply symmetry operations of $\mathbf{G}^{\prime}$ to the shifted pair. If the pair is changed by the symmetry operation, proceed to the next operation: its image has been already rejected. As the cell index also changes once the operations are applied, consider only one of them (for instance, the largest) for the shifted pair to be left, and reject all other pairs generated by $\mathbf{G}^{\prime}$. Since shifted pairs 
can also be mapped onto themselves by certain symmetry operations, the resulting weight factor should be adjusted.

Both algorithms have been implemented in the Gaussian $^{17}$ development version.

\section{B. Formation of the skeleton and full Fock-type matrices}

The reduced lists of shell pairs are processed by the PRISM $^{18}$ algorithm for calculating the two-electron integrals and the Fock matrix formation. This is the most timeconsuming step, especially for HFx, but employing these lists enables a substantial speedup for the entire calculation. The formation of the full Fock matrix from the skeleton matrix is performed straightforwardly according to Eq. (3). As we have already noticed in describing Algorithm 1, an arbitrary symmetry operation transforms the pair $\left(I^{0} J^{g}\right)$ to the pair $\left(I^{\prime h} J^{\prime g^{\prime}}\right)$, therefore the symmetry image of $\mathbf{F}_{I J}^{\mathbf{0 g}}$ is $\mathbf{F}_{I^{\prime} J^{\prime}}^{\mathbf{h}, \mathbf{g}^{\prime}}$ where $\mathbf{h}$ can be different from 0. Due to translational invariance of the Fock matrix $\mathbf{F}_{I J}^{\mathbf{0 g}}=\mathbf{F}_{I J}^{\mathbf{h}, \mathbf{g}+\mathbf{h}}$ and the relation $\mathbf{F}_{I J}^{\mathbf{0 g}}=\mathbf{F}_{J I}^{\mathbf{0},-\mathbf{g}}$ the symmetry contribution from $\mathbf{F}_{I J}^{\mathbf{0 g}}$ is $\mathbf{F}_{I^{\prime} J^{\prime}}^{\mathbf{0}, \mathbf{g}^{\prime}-\mathbf{h}}$, if $I^{\prime} \geq J^{\prime}$, or $\mathbf{F}_{J^{\prime} I^{\prime}}^{\mathbf{0 , h}} \mathbf{\mathbf { g }}$, if $I^{\prime}<J^{\prime}$.

\section{BENCHMARK CALCULATIONS AND COMPUTATIONAL EFFICIENCY}

We have tested our new code against the conventional Gaussian $^{17}$ program for the following systems: C (diamond), $\mathrm{Si}$ (diamond-like structure), ZnS (zinclblend and wurtzite), GaN (wurtzite-type structure), BaS, and BN. The screened hybrid functional of Heyd, Scuseria, and Ernzerhof (HSE) ${ }^{19}$ was employed in all our calculations. All structural parameters, basis sets, and effective core potentials are taken from Ref. 20, where the assessment of the HSE functional for various crystalline structures was reported. For these tests, we have chosen the smallest possible unit cells: two atoms per cell for $\mathrm{C}, \mathrm{Si}$, zincblend, and $\mathrm{BaS}$, four atoms for wurtzite and BN. In most cases, these cells are not conventional crystallographic unit cells as described in Table 2.1.2.1 in Ref. 10, and the set of translational vectors is not left invariant under the point group of the lattice; nevertheless, all operations of the space groups are used since they still comply with the structure. This choice brings in the restrictions we need to apply using algorithms 1 and 2 thus deliberately making the code work in a presumably unfavorable environment. The results are summarized in Table I. We report speedup factors for the PRISM algorithm at the most expensive steps of evaluating near-field Coulomb matrix and HFx as observed during a single iteration of the SCF cycle.

Let us notice that even though the systems chosen for the tests possess high symmetry, the atoms occupy special positions, i.e., are mapped onto themselves by non-trivial operations. Due to the high site-symmetry of such special positions, the resulting speedup factors are noticeably lower than the number of operations used. This observation is in agreement with the earlier findings in Ref. 21.
TABLE I. Speedup factors (SF) are given for the near-field Coulomb (NF J, left-hand side reduction) and HFx (left-hand side reduction (L) and righthand side reduction $(\mathrm{R})$, if available).

\begin{tabular}{lccc}
\hline \hline System & $\begin{array}{c}\text { Space group, } \\
\text { number of operations }\end{array}$ & SF, NF J & $\begin{array}{c}\text { SF, HFx } \\
\mathrm{L} \mid \mathrm{R}\end{array}$ \\
\hline $\mathrm{C}$ & $F d \overline{3} m, 192$ & 9.2 & $6.0 \mid 8.2$ \\
$\mathrm{Si}$ & $F d \overline{3} m, 192$ & 4.2 & $5.3 \mid 5.6$ \\
$\mathrm{BaS}$ & $F m \overline{3} m, 192$ & 2.0 & $2.4 \mid 3.4$ \\
$\mathrm{ZnS}$ (zincblend) & $F \overline{4} 3 m, 96$ & 3.7 & $2.8 \mid 3.5$ \\
$\mathrm{ZnS}$ (wurtzite) & $P 6_{3} m c, 12$ & 3.0 & $2.6 \mid \mathrm{n} / \mathrm{a}$ \\
$\mathrm{GaN}$ & $P 6_{3} m c, 12$ & 3.3 & $2.8 \mid \mathrm{n} / \mathrm{a}$ \\
$\mathrm{BN}$ & $P 6_{3} / m m c, 24$ & 4.3 & $3.9 \mid \mathrm{n} / \mathrm{a}$ \\
\hline \hline
\end{tabular}

We have also performed a simple test to evaluate the importance of non-symmorphic group operations in the resulting speedup factors. Using the same settings for diamond as above and the STO-3G basis set, we reduce the symmetry from $F d \overline{3} m$ (Group No. 227, 192 operations) to $F \overline{4} 3 m$ (Group No. 216, 96 operations). In the latter calculation, evaluation of the HFx contribution with the right-hand side reduction is slower by a factor of 1.5 compared to the former.

If atomic positions are of high multiplicity, the system is expected to display more significant reduction of the computational time. In this work, we apply our technique also to chiral single-walled carbon nanotubes (SWNT) described by non-symmorphic groups. Similar calculations have been reported in the literature using the CRYSTAL code (Refs. 22-25). In Table II we report our findings on several species. The geometries were generated using the TubeGen ${ }^{26}$ program. The symmetry properties of the chiral tubes $(7,4)$ (Fig. 3) and (16,7) (Fig. 4) are taken from Ref. 27. These species are characterized by a general type of screw axis $C_{m / n}$ : the group generating operation consists of a rotation by $\phi=2 \pi n / m$ and a shift by $1 / \mathrm{m}$ of the primitive translation vector. For the $(7,4)$ tube we use the same basis set as for diamond, while for numerical experiment with the $(16,7)$ tube we restrict ourselves to the minimal STO-3G set. Speedup factors are given, as above, for the PRISM algorithm computing the near-field Coulomb and HFx parts per one SCF iteration. Results in Table II show that substantial speedup factors between $9 \mathrm{X}$ and $70 \mathrm{X}$ for the near-field Coulomb and between $9 \mathrm{X}$ and $57 \mathrm{X}$ for HFx can be achieved for these carbon nanotubes.

TABLE II. Speedup factors (SF) for single-walled carbon nanotubes. Nearfield Coulomb (NF J) and HFx. Left-hand side reduction only.

\begin{tabular}{lccr}
\hline \hline System & $\begin{array}{c}\text { Symmetry group, } \\
\text { number of operations }\end{array}$ & SF, NF J & SF, HFx \\
\hline$(6,0)$ & $D_{6 h}, 24$ & 9.3 & 8.9 \\
$(15,0)$ & $D_{15 h}, 60$ & 22.0 & 21.3 \\
$(7,4)$ & $C_{62 / 17}, 62$ & 28.0 & 29.0 \\
$(16,7)$ & $C_{278 / 121}, 278$ & 69.7 & 56.7 \\
\hline
\end{tabular}




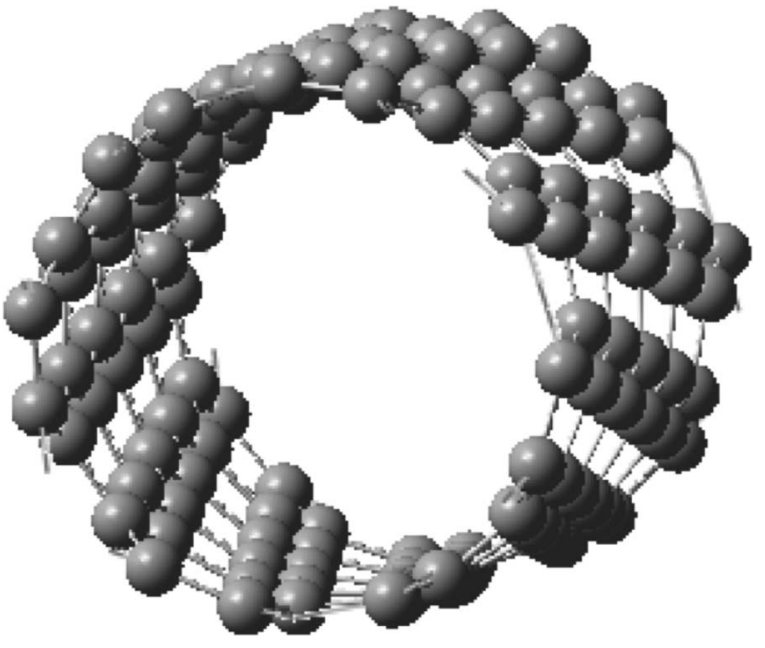

FIG. 3. (7,4) SWNT.

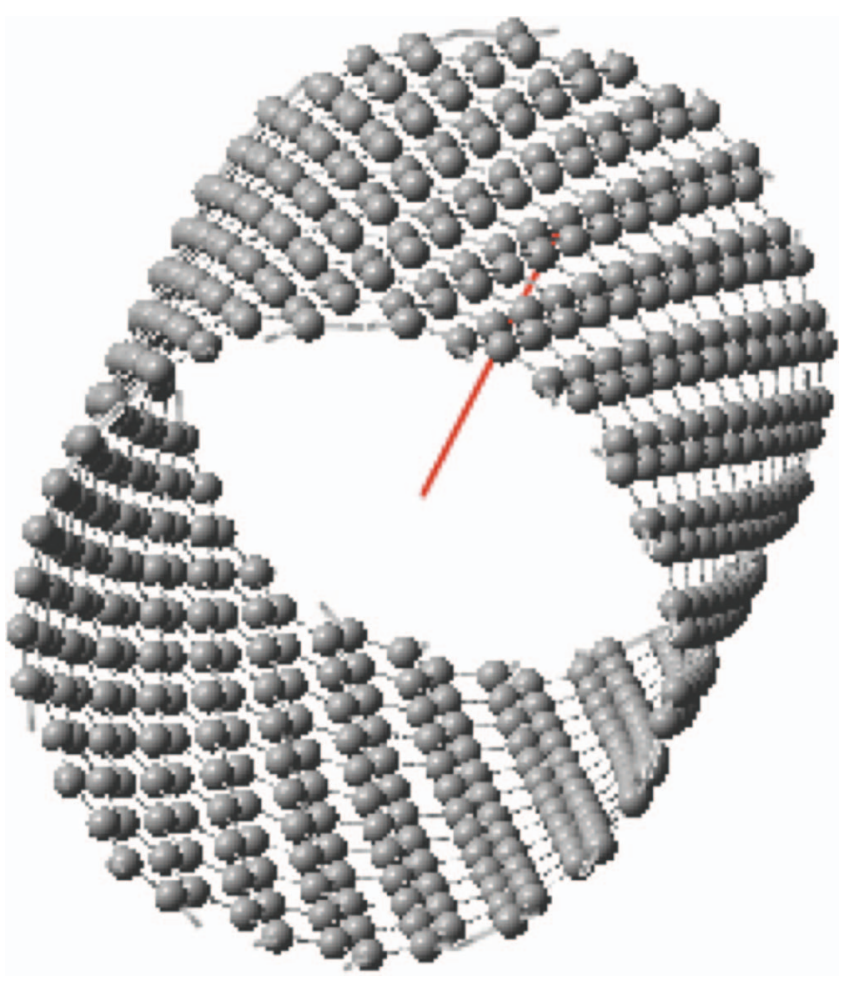

FIG. 4. (16,7) SWNT.

\section{CONCLUSIONS}

Applying full space group symmetry to SCF calculations with $\mathrm{PBC}$ enables significant reduction of the number of explicitly evaluated two-electron integrals and substantial speedup of the generally slowest step of the calculation. Quasi-1D systems of high symmetry and multiplicities of the atomic positions display largest speedup factors exceeding one order of magnitude. Systems periodic on all three dimen- sions, though having high symmetry, tend to have low multiplicities of the atomic positions, thus leading to speedup factors much smaller than the number of operations, but still noticeably reducing the cost of evaluating the two-electron integrals for exchange.

\section{ACKNOWLEDGMENTS}

This work was supported by DOE, Office of Basic Energy Sciences, Heavy Element Chemistry program, under Grant No. DEFG02-04ER15523. A.A.R. is grateful to Ireneusz Bulik and Roman Schutski for helpful advice and fruitful discussions. G.E.S. is a Welch Foundation Chair (C-0036).

${ }^{1}$ G. E. Scuseria, J. Phys. Chem. A 103, 4782 (1999).

${ }^{2}$ A. D. Becke, J. Chem. Phys. 98, 1372 (1993).

${ }^{3}$ B. G. Janesko, T. M. Henderson, and G. E. Scuseria, Phys. Chem. Chem. Phys. 11, 443 (2009).

${ }^{4}$ T. M. Henderson, J. Paier, and G. E. Scuseria, Phys. Status Solidi B 248, 767 (2011).

${ }^{5}$ K. N. Kudin and G. E. Scuseria, Phys. Rev. B 61, 16440 (2000).

${ }^{6}$ R. E. Stratmann, G. E. Scuseria, and M. J. Frisch, Chem. Phys. Lett. 257, 213 (1996).

${ }^{7}$ V. R. Saunders, C. Freyria-Fava, R. Dovesi, L. Salasco, and C. Roetti, Mol. Phys. 77, 629 (1992).

${ }^{8}$ M. C. Strain, G. E. Scuseria, and M. J. Frisch, Science 271, 51 (1996).

${ }^{9}$ A. F. Izmaylov, G. E. Scuseria, and M. J. Frisch, J. Chem. Phys. 125, 104103 (2006).

${ }^{10} \mathrm{Th}$. Hahn, International Tables for Crystallography. Volume A: SpaceGroup Symmetry (Springer, Dordrecht, 2005).

${ }^{11}$ V. Kopský and D. B. Litvin, International Tables for Crystallography. Volume E: Subperiodic Groups (Kluwer Academic Publishers, 2002).

${ }^{12}$ M. Dupuis and H. F. King, Int. J. Quantum Chem. 11, 613 (1977).

${ }^{13}$ R. Dovesi, Int. J. Quantum Chem. 29, 1755 (1986).

${ }^{14}$ C. Pisani, R. Dovesi, and C. Roetti, Hartree-Fock Ab Initio Treatment of Crystalline Systems, Lecture Notes in Chemistry Vol. 48 (Springer-Verlag, 1988), p. 96.

${ }^{15}$ B. A. DeAngelis, R. E. Newnham, and W. B. White, Am. Mineral. 57, 255 (1972).

${ }^{16}$ R. A. Evarestov and V. P. Smirnov, Site Symmetry in Crystals: Theory and Applications, Springer Series in Solid-State Sciences Vol. 108 (SpringerVerlag, 1993).

${ }^{17}$ M. J. Frisch, G. W. Trucks, H. B. Schlegel et al., Gaussian Development Version, Revision H.11, Gaussian, Inc., Wallingford, CT, 2010.

${ }^{18}$ P. M. W. Gill and J. A. Pople, Int. J. Quantum Chem. 40, 753 (1991).

${ }^{19}$ J. Heyd, G. E. Scuseria, and M. Ernzerhof, J. Chem. Phys. 118, 8207 (2003).

${ }^{20}$ M. J. Lucero, T. M. Henderson, and G. E. Scuseria, J. Phys.: Condens. Matter 24, 145504 (2012).

${ }^{21}$ C. M. Zicovich-Wilson and R. Dovesi, Int. J. Quantum Chem. 67, 311 (1998).

${ }^{22}$ P. D'Arco, Y. Noel, R. Demichelis, C. M. Zicovich-Wilson, and R. Dovesi, J. Chem. Phys. 131, 204701 (2009).

${ }^{23}$ Y. Noel, P. D'Arco, R. Demichelis, and R. Dovesi, J. Comput. Chem. 31, 855 (2010).

${ }^{24}$ R. Demichelis, Y. Noel, P. D'Arco, L. Maschio, R. Orlando, and R. Dovesi, J. Mater. Chem. 20, 10417 (2010).

${ }^{25}$ K. E. El-Kelany, M. Ferrabone, M. Rérat, Ph. Carbonnière, C. M. ZicovichWilson, and R. Dovesi, Phys. Chem. Chem. Phys. 15, 13296 (2013).

${ }^{26}$ TubeGen 3.4 (web-interface, http://turin.nss.udel.edu/research/ tubegenonline.html), J. T. Frey and D. J. Doren, University of Delaware, Newark, DE, 2011.

${ }^{27}$ R. A. Jishi, M. S. Dresselhaus, and G. Dresselhaus, Phys. Rev. B 47, 16671 (1993). 